\title{
Influence of Surface Roughness and Temperature on Wheel / Rail Adhesion in Wet Conditions
}

\author{
Hiraku TANIMOTO Hua CHEN \\ Track Dynamics Laboratory, Railway Dynamics Division
}

\begin{abstract}
A series of experiments on the wheel/rail traction coefficient were carried out with a twin disc rolling contact machine by varying the surface roughness and the temperature of the wheel and rail discs, and using sprayed water, for the purpose of obtaining fundamental knowledge about how to prevent wheels slipping during driving and wheel sliding during braking. The results showed that the maximum traction coefficient value occurred in the range of 1 to $3 \mu \mathrm{m}$ of the combined roughness under any temperature conditions, and its value increased to 0.4 with increases in temperature.
\end{abstract}

Keywords: wheel/ rail, adhesion, surface roughness, temperature, wet

\section{Introduction}

In order to obtain fundamental insight into factors influencing wheel slipping in driving and sliding in braking, those elements deemed to have most impact on wheel/ rail adhesion, i.e. wheel/rail surface roughness, and the temperature of wheel, rail and water, were studied through experiments described in this paper.

Rain can lead to wheel slipping and sliding, due to the presence of water on the wheel/rail surface [1]. Adhesive blocks offer an effective countermeasure against wheel sliding, and have three functions; firstly of cleaning, by removing substances on the wheel tread such as dust, oil and oxide film, secondly of attaching hard particles on the wheel tread, and thirdly of creating wheel surface roughness. With respect to the third function, Ohyama et al. [2] studied the influence of surface roughness of the wheel on wheel/rail adhesion and reported that adhesion increased with surface roughness of the wheel.

Chen et al. [3], [4] studied the relationship between adhesion and the various temperatures of the sprayed water and reported that the adhesion obtained through experiments and computational calculation increased with the water temperature.

On the other hand, some studies referring to the mechanism underlying how adhesion decreases in wet conditions compared to dry conditions, reported that a small volume of ferric oxide paste generated by the chemical reaction between steel and water reduced adhesion [5], [6] and that the action of fluid dynamics on the wheel/rail surface because of water, reduced the adhesive force [3]. The former mechanism can explain phenomenon [7] characterized by reduced adhesion of a first train, heavy fog or when rain begins to fall while the latter accounts for reduced adhesion on trains running at high speed [8].

Both mechanisms are rational, and there is a possibility that both effects occur simultaneously. The phenomena display features of boundary lubrication and elastohydrodynamic lubrication. Boundary lubrication describes the lubricous condition induced by the boundary film generated by the adsorption of fluid molecules on the solid surface with the van der Waals' force and the chemical bond. Elastohydrodynamic lubrication corresponds to the lubricated condition created by the fluid film which occurs due to the fluid dynamics and the elastic deformation of contacting bodies. Boundary lubrication is intricately interrelated with physical and chemical phenomena. Few studies on boundary lubrication relating to friction between metal and water have been made [9], because cases in which metals are rubbed with water as a lubricant in practice are unusual. Over the past 20 years, analytical methods using molecular dynamic simulation have been established to study the mechanics of traction devices, but the reported studies [10], [11] dealt with the phenomenon of traction devices and high-efficiency oils. Wheel/rail phenomena are neither obvious physically nor chemically. Therefore, it is still difficult to resolve wheel/rail adhesion problems in wet conditions using molecular dynamic simulation.

This study describes experiments using an experimental machine focusing on the effects of surface roughness and temperature which directly affect boundary film and fluid film formation. The traction coefficients were measured by varying the surface roughness of the wheel disc and the rail disc, the temperature of the wheel, rail and sprayed water in a wider range than that of reported data in past studies.

\section{Experimental Method}

\subsection{Machine}

A twin disc rolling contact machine and water spray device were used for the experiments. Figure 1(a) shows the mechanical composition of the machine. It has two disc-shaped rigs, namely, wheel and rail discs, an AC motor for driving the wheel disc, an eddy current brake connected to the rail disc axis, a hydraulic system for loading vertical force, and measurement systems of rotational velocity, contact force and torque of the wheel disc. Figure 1(b) shows the shape and dimensions of the two discs. The wheel disc was made of JIS SSW-Q1R and the rail disc was cut and processed from a standard JIS $60 \mathrm{~kg}$ rail. The water spray device was incorporated into the machine in order to spray the water into the contact zone of the wheel and the rail discs. A jet of water at a given temperature was sprayed 


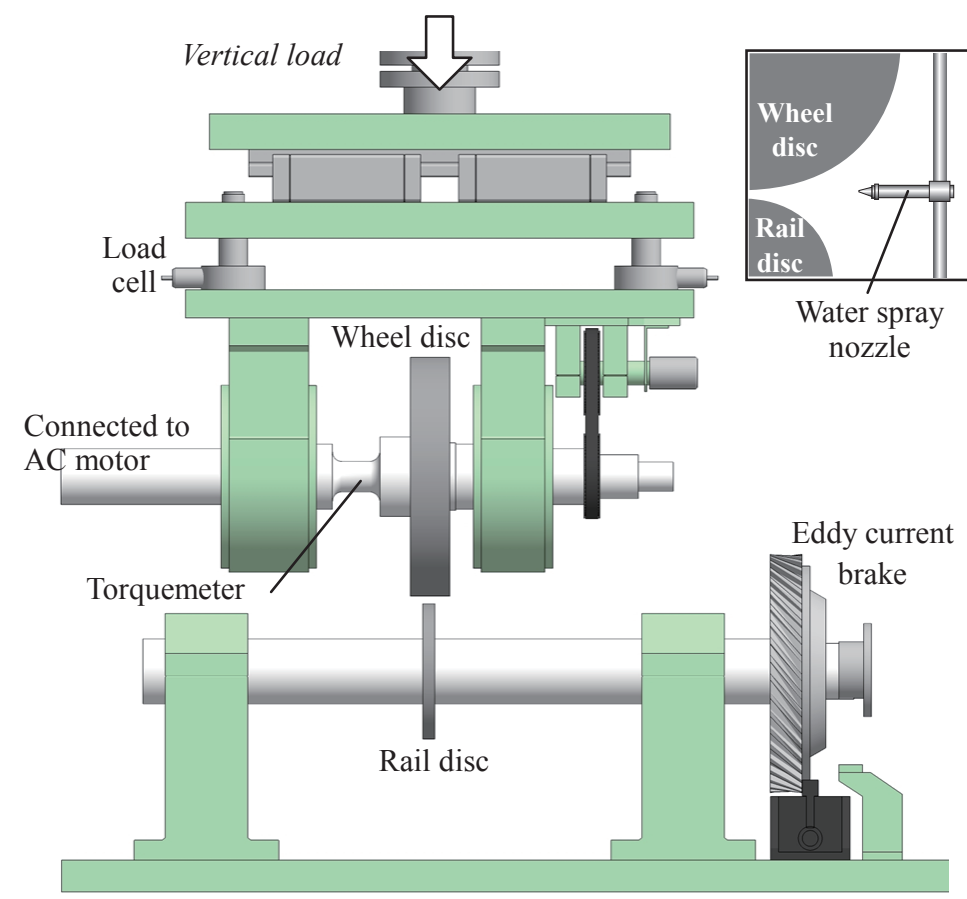

(a) Schematic image of the machine

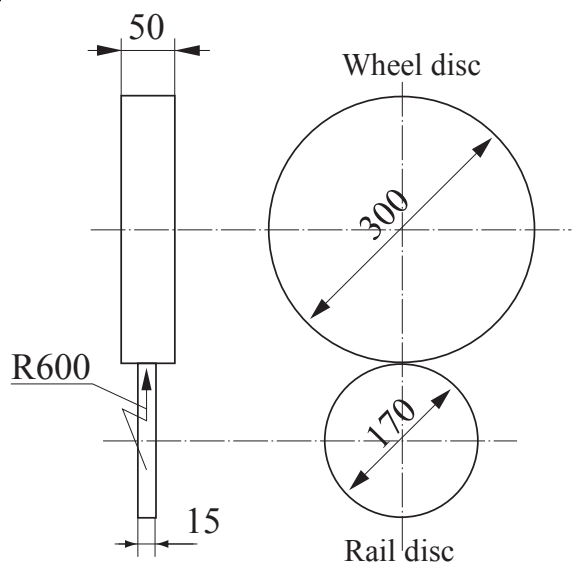

(b) Shape and dimensions of two discs

Fig. 1 Twin disc rolling contact machine

from the water tank by compressed air. The nozzle used to spray water had a circular cylinder of $1.2 \mathrm{~mm}$ in the bore diameter and the distance from the tip of the nozzle to the contact point of the two discs was $170 \mathrm{~mm}$.

\subsection{Procedures}

The experiment was conducted as follows: first, the roughness of the contact surface of the two discs was adjusted with abrasive paper and measured by a stylus type surface roughness measuring instrument. Second, the temperature of the two discs and water were adjusted with the condition of "Heated," "Room temperature" or "Cooled." The temperature of the laboratory was maintained at room temperature with air conditioning equipment. The adhesion experiments were then conducted using this machine after the temperature of the discs and water was adjusted.

\subsection{Conditions}

The experimental conditions are shown in Table 1. The control patterns of the velocity and the load in the adhesion experiments are shown in Fig.2. After bringing the wheel disc into contact with the rail disc, and loading the vertical force, the wheel disc was rotated by the motor while the rail disc was rotated by the traction between two discs. Water was sprayed after acceleration of the discs. The brake force on the rail disc was increased with a slip ratio of $0 \%$ to $20 \%$. The slip ratio is defined as the value obtained by dividing the difference between the wheel disc velocity and the rail disc velocity, by the mean value of the wheel velocity and the rail disc velocity. When the slip ratio reached $20 \%$ through braking on the rail disc, the rotation
Table 1 Experimental conditions

\begin{tabular}{c|c}
\hline $\begin{array}{c}\text { Vertical load } \\
\text { (Max. Hertz pressure) }\end{array}$ & $\begin{array}{c}4 \mathrm{kN} \\
(780 \mathrm{MPa})\end{array}$ \\
\hline Velocity & $100 \mathrm{~km} / \mathrm{h}$ \\
\hline Slip ratio & $0 \sim 20 \%$ \\
\hline Sprayed water & $10 \mathrm{ml} / \mathrm{s}$ \\
\hline $\begin{array}{c}\text { Water velocity } \\
\text { at nozzle tip }\end{array}$ & $8.4 \mathrm{~m} / \mathrm{s}$ \\
\hline
\end{tabular}

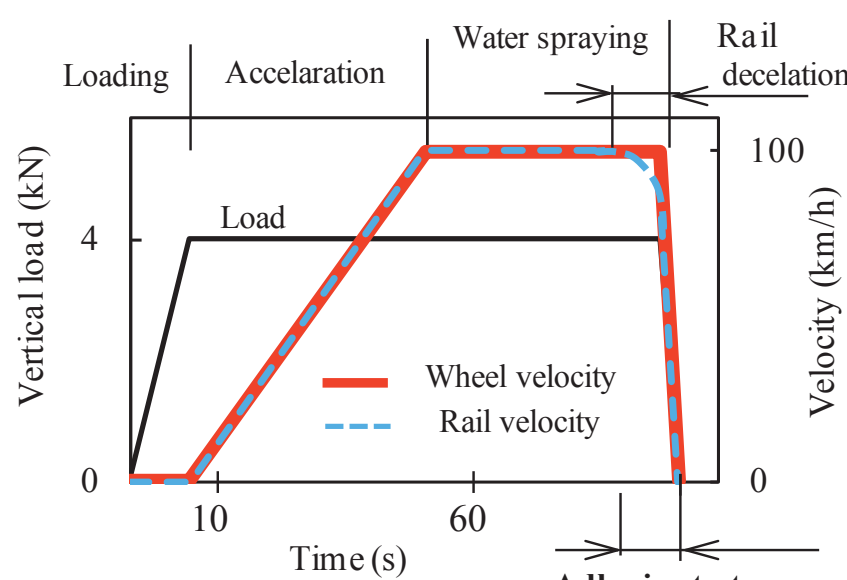

Adhesion test

Fig. 2 Control of load and velocity

of the wheel disc and the rail disc automatically stopped and unloaded. The relationship between the slip ratio and the traction coefficient which is the value of the tangential force divided by the vertical force can be obtained by the vertical load, the wheel torque and the rotational velocity of 


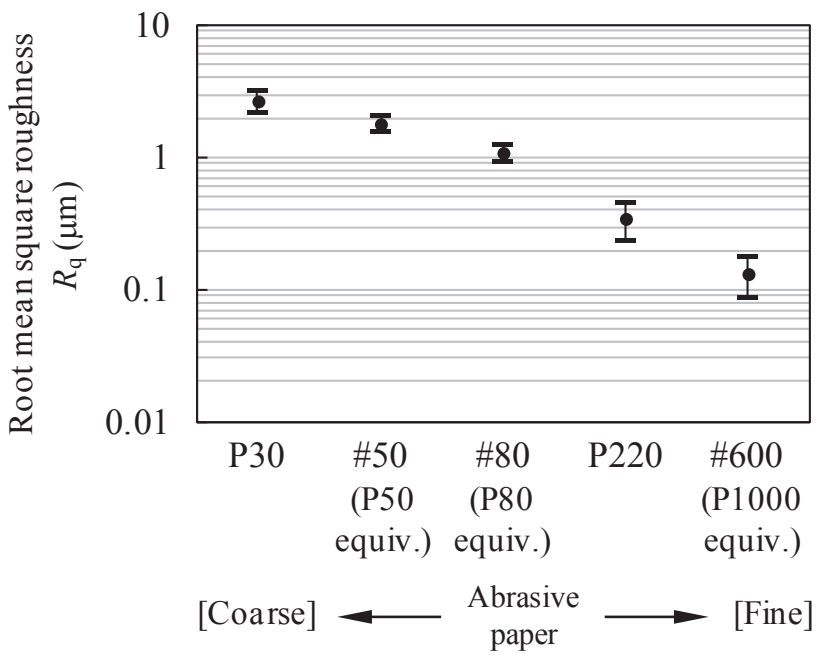

Fig. 3 Roughness in accordance with the abrasive paper numbers

the wheel and rail discs while a brake force was being applied to the rail disc. Each value was measured at $200 \mathrm{~Hz}$.

A vertical load of $4 \mathrm{kN}$ was set, taking into consideration the fact that the contact pressure exerted on the two discs was about $800 \mathrm{MPa}$, the same as the contact pressure between the actual wheel of $860 \mathrm{~mm}$ in diameter with a conical profile tread, and the rail which has a head radius of $600 \mathrm{~mm} .100 \mathrm{~km} / \mathrm{h}$ is the maximum velocity of the experimental machine. It was considered that the maximum slip ratio $20 \%$ would not cause excessive damage to the wheel and rail discs. The volume of sprayed water and its outflow velocity were set to the maximum possible value for the water tank.

In order to produce accurate roughness on the two discs using each numbered piece of abrasive paper, roughness-producing devices for the wheel and the rail discs were developed. The roughness given by line-like traces in the direction of the rotation of the discs was produced by $78 \mathrm{~N}$ of the load and $3 \mathrm{~km} / \mathrm{h}$ of the circumferential velocity. The roughness values produced by each kind of abrasive paper are shown in Fig. 3. The vertical axis is the root mean square, RMS, roughness which is determined as follows:

$$
R_{q}=\sqrt{\frac{1}{l} \int_{0}^{l} f(x)^{2} d x}
$$

where $l$ is an evaluated length of $4 \mathrm{~mm}, x$ is the length in the measurement direction and $f(x)$ is the curve of the surface profile. $R_{\mathrm{q}}$ is equivalent to the standard deviation of the surface roughness. While in most of these experiments, the roughness of the wheel disc and that of the rail disc were given by abrasive paper with the same number, some experiments were additionally conducted using abrasive paper with different numbers between the wheel and the rail disc. The data of the roughness of the discs was evaluated by the combined roughness:

$$
\sigma=\sqrt{R_{q w}{ }^{2}+R_{q r}{ }^{2}}
$$

where $R_{\mathrm{qw}}$ and $R_{\mathrm{qr}}$ are the RMS roughness of the wheel and the rail discs, respectively.

The conditions of the temperature are shown in Table 2 and the dispersions of the temperature of the three conditions are shown in Fig.4. For the "Heated" conditions a gas burner was used to heat the side faces of the rotating two discs to avoid the heat affecting each contact surface. For the "Cooled" conditions dry ice particles were pressed to the contact surfaces with rotating discs.

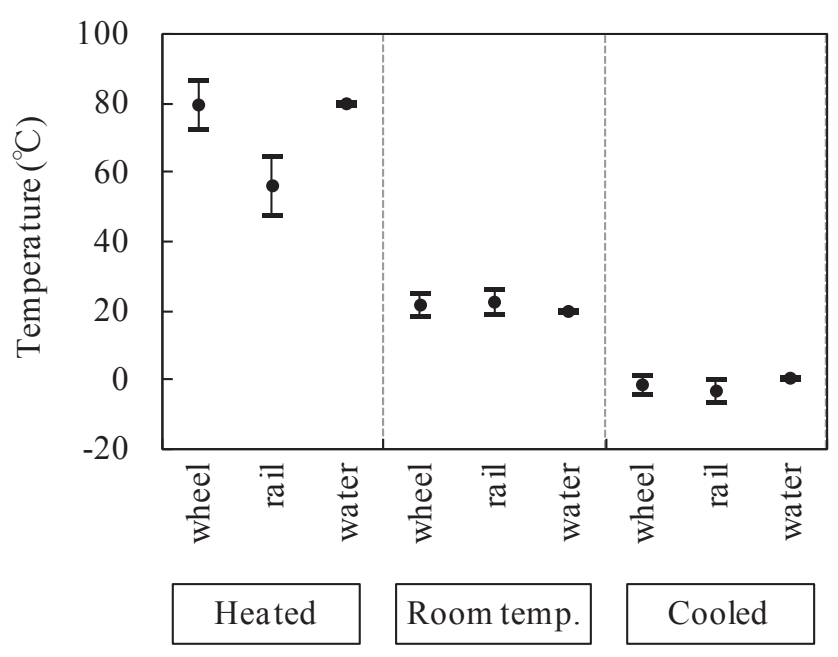

Fig. 4 Temperature in accordance with each condition

Table 2 Temperature conditions

\begin{tabular}{c|c|c|c|c|c|c}
\hline Condition & A & B & C & D & E & F \\
\hline $\begin{array}{c}\text { Conducted experiment } \\
\text { number }\end{array}$ & 4 & 2 & 7 & 12 & 2 & 9 \\
\hline Wheel & Heated & Heated & R. T. & R. T. & R. T. & Cooled \\
Rail & Heated & R. T.* & R. T. & R. T. & R. T. & Cooled \\
Water & Heated & Cooled & Heated & R. T. & Cooled & Cooled \\
\hline
\end{tabular}

*R. T.: Room Temperature 


\section{Results}

An example of the experimental results is shown in Fig.5. The relationship between the slip ratio and the traction coefficient was calculated from the twenty-point moving-average of the rotational velocity and the load. In this paper, "adhesion coefficient" is defined as the local maximum value of the traction coefficient around a slip ratio of $0-1 \%$.

Figure 6 shows all the experimental results giving the adhesion coefficient versus the combined roughness. The dashed lines in Fig. 6 show the changing tendencies of the adhesion coefficient under temperature conditions A, C, D and $\mathrm{F}$, and the asterisk indicates re-adhesion; the details are shown in section 4.2. From Fig.6, it can be seen:

(1) The adhesion coefficients have a local maximum or nearly constant value at 1 - $3 \mu \mathrm{m}$ combined roughness.

(2) The adhesion coefficients grow as temperature of the wheel disc increases, in the range where the adhesion coefficients have a local maximum or nearly constant value.

(3) In the region of less than $1 \mu \mathrm{m}$ combined roughness, the adhesion coefficients decrease and the influence of the temperature falls with the decrease of the combined roughness.

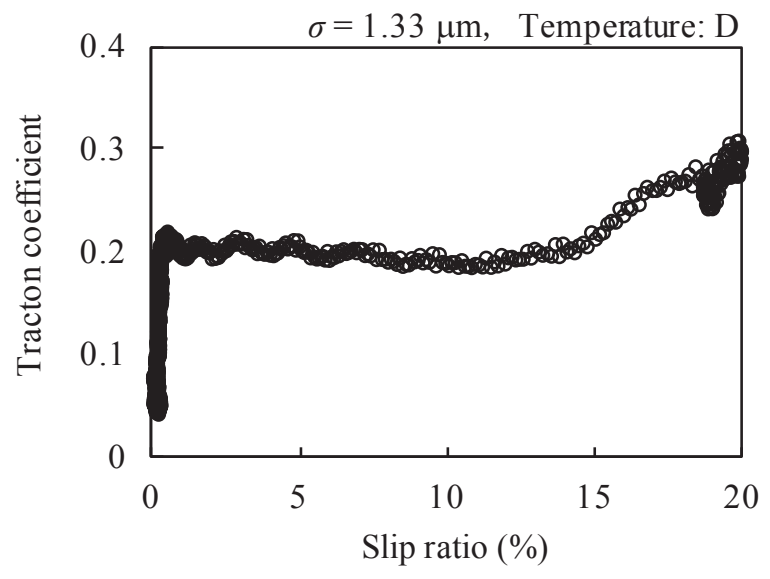

Fig. 5 Example of experimental results

\section{Discussions}

\subsection{Film parameter}

The fluid film with the flow of lubricant can be generated in the moving Hertz contact area. The thickness of the fluid film was calculated by the Elastohydrodynamic Lubrication, EHL theory [12]. The theory is composed of three equations, an elasticity equation for two elastic bodies, a Navier-Stokes equation and an equation of the state of fluid viscosity. The shape of the fluid film and the pressure distribution in the contact area can be determined by the EHL theory. However, EHL theory holds true only if the lubricant in the contact area moves in laminar flow. Therefore, the limitation of the theory, i.e., the boundary between the film formation and fracture, cannot be solved by the theory alone. Johnson et al.[13] studied the influence of the roughness on the EHL film formation, comparing the theoretical equation and the experimental results by means of the film parameter $\Lambda=h / \sigma$, where $h$ is the minimum EHL film thickness with the smooth contact surfaces and $\sigma$ is the combined roughness. According to their results, the contact surfaces are almost always in contact with each other at $\Lambda<1$ and almost always separated from each other at $\Lambda>3$. In their research, the influence of the traction was not considered, but Muraki and Kimura [14] reported on the relationship between $\Lambda$ and the traction coefficient which corresponds to the adhesion coefficient in this paper. According to their research on the change of the traction coefficient against the surface roughness, the traction coefficient reaches its largest value and the wear volume increases dramatically at $\Lambda=1 \sim 2$ when decreasing $\Lambda$ from around 40. Muraki and Kimura used mineral oil as the lubricant, but the relationship between the condition of the contact surface and $\Lambda$ is controlled mainly by the geometry of the surfaces. Therefore, even in the case of water, the same relationship between the traction coefficient and $\Lambda$ as the case of mineral oil is established.

As the parameter $\Lambda$ can serve as a useful index for understanding phenomena on the contact surfaces, the authors calculated $\Lambda$ using the experimental results obtained in this study. First, the minimum film thickness under each temperature condition should be estimated. The equation of Hamrock-Dowson [15] and the viscosity of the water
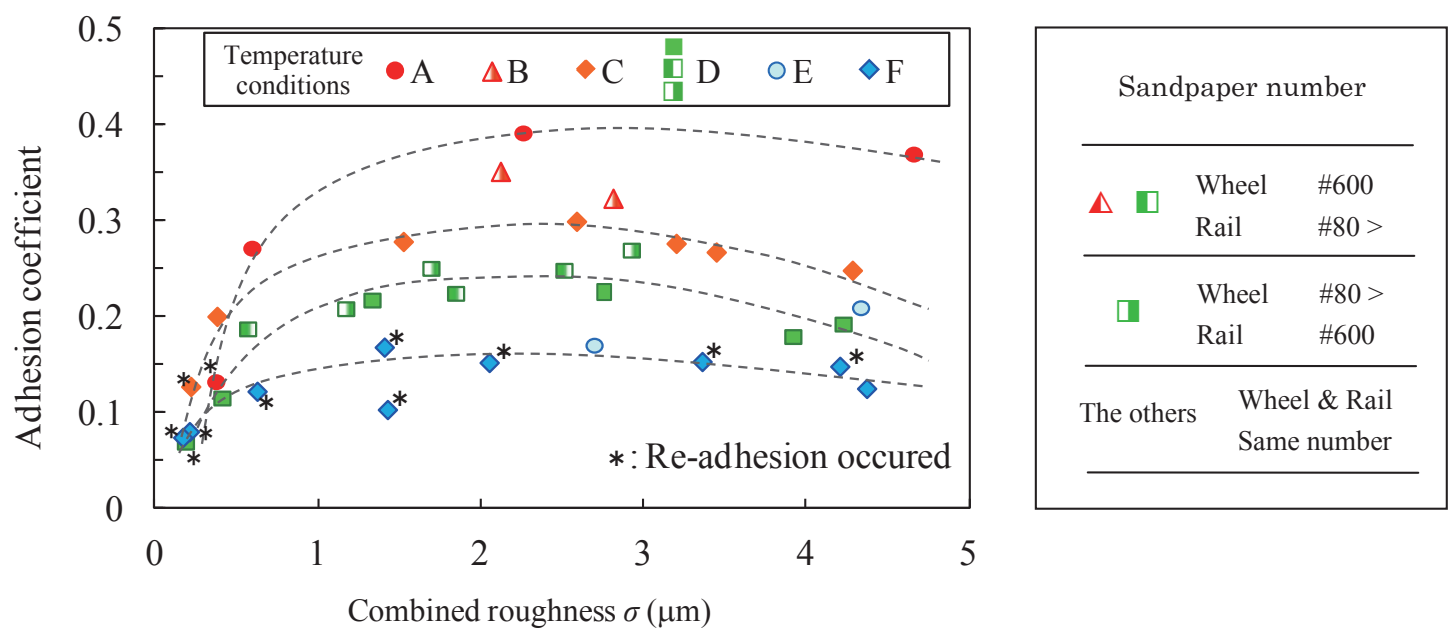

Fig. 6 Adhesion coefficient against combined roughness 
Table 3 Film thickness and used values of the parameters

\begin{tabular}{cccc}
\hline $\begin{array}{c}\text { Water temperature } \\
\left({ }^{\circ} \mathrm{C}\right)\end{array}$ & $\begin{array}{c}\text { Viscosity under } \\
\text { atmospheric pressure } \\
(\mathrm{mPa} \cdot \mathrm{s})\end{array}$ & $\begin{array}{c}\text { Viscosity-pressure } \\
\text { coefficient } \\
\left(\mathrm{x} 10^{-10} \mathrm{~Pa}^{-1}\right)\end{array}$ & $\begin{array}{c}\text { Minimum film } \\
\text { thickness } \\
(\mu \mathrm{m})\end{array}$ \\
\hline 2.2 & 1.66 & 3.9 & 1.3 \\
\hline 20 & 1.02 & 6.5 & 1.2 \\
\hline 75 & 0.38 & 6.1 & 0.6 \\
\hline
\end{tabular}

by Bett and Cappi [16] were applied for the estimation of the film thickness. The estimated values based on the size and shape of the discs, and the conditions of this study are shown in Table 3 . The water temperature, the viscosity under the atmospheric pressure and the viscosity-pressure coefficient are also shown in Table 3. Data from literature on viscosity at the temperatures nearest to the conditions of these experiments were referred to here. Therefore, the water temperature in Table 3 does not quite tally with the experiment temperatures.

The parameter $\Lambda$ was calculated from the minimum thickness and roughness. The relationship between adhesion coefficients and $\Lambda$ 's are shown in Fig.7. $\Lambda$ is less than $3 \mu \mathrm{m}$ except for three plotted data at the right in Fig. 7 . Therefore, the EHL film was not formed in the experiments except for the three cases, and that the surfaces of the discs could be in contact with each other directly or through the intermediary of a boundary film in the region of less than $1 \mu \mathrm{m}$ of the combined roughness.

The result (3) in section 4.1 can be explained by the above consideration. The region of less than $1 \mu \mathrm{m}$ of the combined roughness corresponds to the region of more than around 1 in film parameter shown in Fig.7. In this range, the adhesion coefficient decreases with the decrease of the combined roughness by the influence of the fluid film which is generated in this range. On the other hand, the reason for the result of (1) in section 4.1 cannot be explained only by the above consideration, but the adhesion coefficient may decrease due to the effect of the fluid trapped in concave areas of the surfaces with the increased roughness as is inferred from Kataoka [17] which reports that the frictional force between processing steel and an edge tool decreased because of lubricant in micro pools on the surface. However, the events between the actual wheel and rail cannot necessarily be evaluated quantitatively by the above consideration. The understanding of the phenom-

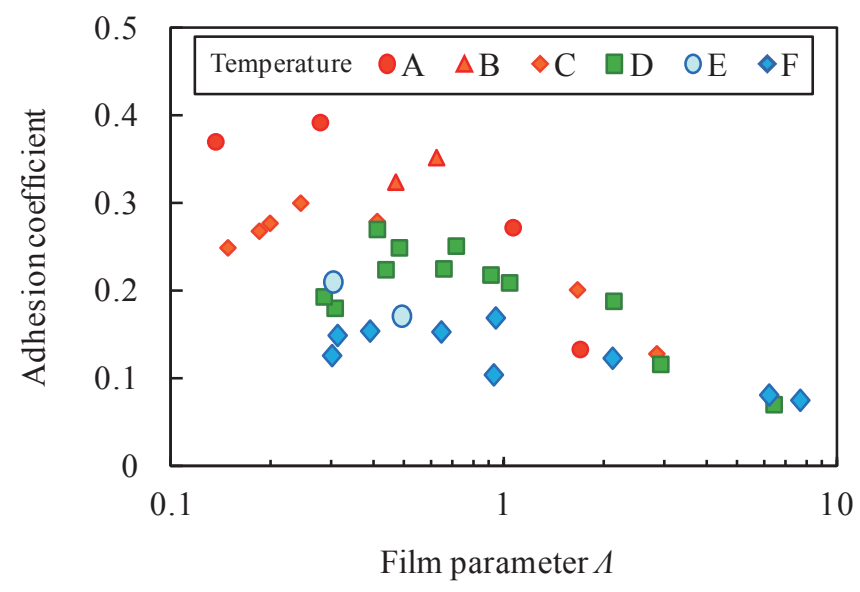

Fig. 7 Adhesion coefficient against film parameter enon of the experiment is needed first in order to elucidate wheel/rail adhesion.

\subsection{Re-adhesion}

Re-adhesion occurred during the adhesion experiments marked $*$ in Fig.6. An example of re-adhesion is shown in Fig.8. The slip ratio at the time of the occurrence of the readhesion varies a great deal in the range between $13 \sim 20$ $\%$ and there is no definite correlation between the slip ratio and the experimental conditions except for a slip ratio of 5.8 $\%$ under the temperature condition A with abrasive papers \#600 for both discs.

A possible cause of the re-adhesion is the rise in temperature of the contact surfaces from an increase in slip velocity. The temperature of the frictional surface generally increases with the increase in slip velocity. The reduction and/or the fracture of the fluid film and the boundary film occur with a decrease of the viscosity of the fluid when the temperature rises, causing metallic adhesion in the contact areas and an increase in plasticity. However, the detailed mechanisms are unclear and this type of the re-adhesion might be a unique phenomenon of the experimental machine because the temperature of the contact surfaces of the discs rise easily because of repetitive contact in a short time and the small volumes involved, compared to actual wheels and rails.

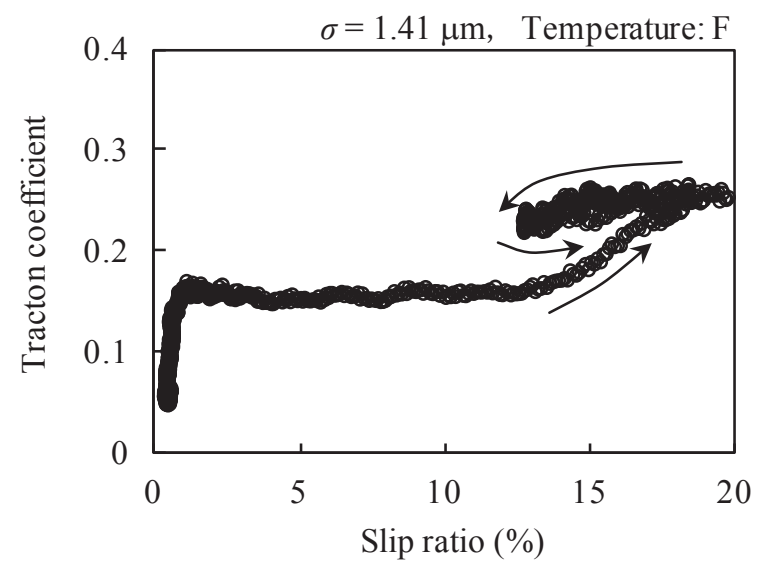

Fig. 8 Example of experimental result where re-adhesion occurred

\section{Conclusions}

Adhesion experiments with a twin disc rolling contact machine were conducted in order to investigate the influence of roughness and temperature on wheel/rail adhesion. The experiment consisted of various combinations of the following factors; $4 \mathrm{kN}$ of the vertical load, $100 \mathrm{~km} / \mathrm{h}$ of 
the experiment velocity, $0.1 \sim 3 \mu \mathrm{m}$ of root mean square roughness and $0 \sim 80{ }^{\circ} \mathrm{C}$ of temperature of the wheel, rail disc and water. The results obtained in this study can be summarized as follows:

(1) Adhesion coefficients have a local maximum or almost constant values at $1 \sim 3 \mu \mathrm{m}$ combined roughness

(2) Adhesion coefficients grow as the temperature of the wheel increases, in the range where adhesion coefficients have a local maximum or almost constant values.

(3) The influence of temperature falls with the decrease in combined roughness in the region of less than $1 \mu \mathrm{m}$ combined roughness

(4) Re-adhesion which is a phenomenon characterized by the dramatic increase of the traction coefficient occurred in some experimental conditions

\section{References}

[1] Nagase, K., "Study of adhesion between the rails and running wheels on main lines," Proc. Inst. Mech. Engrs. Part F, Vol.203, No.16, pp.33-43, 1989.

[2] Ohyama, T., "Tribological studies on adhesion phenomena between wheel and rail at high speeds," Wear, Vol. 144, No.2, pp. 263-275, 1991.

[3] Chen, H., Ban, T., Ishida, M., Nakahara, T., "Adhesion between rail/wheel under water lubricated contact," Wear, Vol.253, No.1, pp.75-81, 2002.

[4] Chen, H., Ban, T., Ishida, M., Nakahara, T., "Experimental investigation of influential factors on adhesion between wheel and rail under wet conditions," Wear, Vol.265, No.9, pp.1504-1511, 2008.

[5] Beargley, T. M., McEwen, I. J., Pritchard, C., "Wheel/ Rail adhesion - The influence of railhead debris," Wear, Vol.33, No.1, pp.141-152, 1975.

[6] Beargley, T. M., Pritchard, C., "Wheel/Rail adhesion -The overriding influence of water," Wear, Vol.35,
No.2, pp.299-313, 1975.

[7] Jenks, C. W., "Improvement methods for increasing wheel/rail adhesion in the presence of natural contaminants," Transit Cooperative Research Program, Research Results Digest, No.17, 1997.

[8] Maruyama, H. et al., "Adhesion and Its Control in High Velocity Railway Cars," Junkatsu, Vol. 25, No. 1, pp. 9-14, 1980 (in Japanese).

[9] Uchidate, M. et al., "Tribological Properties of Stainless under Water Lubricated Conditions," Tribologist, Vol. 49, No. 2, pp. 173-180, 2003 (in Japanese).

[10]Cui, S. T., Cummings, P. T., Cochran, H. D., "Molecular dynamics simulation of the rheological and dynamical properties of a model alkane fluid under confinement," Chem. Phys., Vol.111, No.3, pp.1273-1280, 1999.

[11] Endou, A., Onodera, T., Nara, S., Suzuki, A., Koyama, M., Tsuboi, H., Hatakeyama, N., Takaba, H., Del Carpio C., Kubo, M., Miyamoto, A., "Theoretical study of dynamic behavior of diphenyldisulphide molecule on Fe surface: Novel ultra-accelerated quantum chemical molecular dynamics approach," Tribol. Online, Vol.3, No.5, pp.280-284, 2008.

[12]Dowson, D., Higginson, G. R., "A numerical solution to the elasto - hydrodynamic problem," J. Mech. Engr. Sci., Vol.1, No.1, pp.6-15, 1959.

[13]Johnson, K., Greenwood, J., Poon, S., “A simple theory of asperity contact in elastohydro-dynamic lubrication,"Wear, Vol.19, No.1, pp91-108, 1972.

[14]Muraki, M. and Kimura, Y., "Traction Characteristics of Lubricating Oils (1st Report)," Junkatsu, Vol. 28, No.1, pp.67-74, 1983 (in Japanese).

[15] Hamrock, B., Dowson, D., Ball bearing lubrication, John Wiley \& Sons, 1981.

[16]Bett, K., Cappi, J., "Effect of pressure on the viscosity of water," Nature, Vol.207, pp.620-621, 1965.

[17]Kataoka, S., "Surface Texture of Tools and Workpieces in Plastic Working," Tribologist, Vol. 48, No.9, 2003 (in Japanese).

\section{Authors}

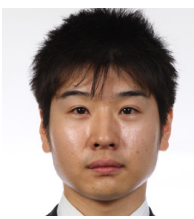

Hiraku TANIMOTO

Researcher, Track Dynamics Laboratory, Railway Dynamics Division Research Areas: Tribology

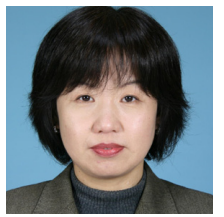

Hua CHEN, Dr. Eng.

Chief Researcher, Track Dynamics

Laboratory, Railway Dynamics Division

Research Areas: Tribology, Wheel/Rail

Contact Mechanics 